\title{
Satisfação no trabalho docente: Uma análise a partir do modelo social cognitivo de satisfação no trabalho e da eficácia coletiva docente
}

Maély Ferreira Holanda Ramos. Universidade Federal do Pará.

Ana Patrícia de Oliveira Fernandez. Instituto Federal de Educação, Ciência e Tecnologia do Pará.

Karla Cristina Nina Furtado. Instituto Federal de Educação, Ciência e Tecnologia do Pará.

Edson Marcos Leal Soares Ramos. Universidade Federal do Pará.

Simone Souza da Costa e Silva. Universidade Federal do Pará.

Fernando Augusto Ramos Pontes. Universidade Federal do Pará.

\section{Resumo}

Considerando que a satisfação no trabalho é um aspecto que tem efeito sobre os estados motivacionais dos professores, faz-se necessário investigar suas implicações na docência. Neste sentido, este estudo tem como objetivo: identificar fatores relacionados à satisfação no trabalho a partir da avaliação das dimensões do Modelo Social Cognitivo de Satisfação no Trabalho (MSCST) e da eficácia coletiva. Para tanto, realizou-se pesquisa numa amostra de 495 professores de uma rede privada de ensino, utilizando abordagem mista (quantitativa-qualitativa). As técnicas estatísticas de análise dos dados foram aplicadas nas informações coletadas referentes à amostra total (495). A análise de conteúdo (abordagem qualitativa) foi aplicada nas respostas abertas de 40 professores pertencentes à amostra. Os resultados indicaram que as crenças de eficácia (autoeficácia e eficácia coletiva) não têm efeito sobre a satisfação no trabalho docente e que outros fatores, como os econômicos, podem estar mais relacionados à satisfação.

Palavras-chave: satisfação no trabalho; professores; eficácia coletiva.

\begin{abstract}
The teacher job satisfaction: An analysis from the social cognitive model of job satisfaction and collective efficacy from teachers. Whereas job satisfaction is an aspect that has an effect on the motivational states of teachers, it is necessary to investigate its implications for teaching. Thus, this study aims to: identify factors related to job satisfaction based on the assessment of the dimensions of the Social Cognitive Model of Job Satisfaction (MSCST) and collective efficacy. For this, research was conducted on a sample of 495 teachers of private schools using mixed approach (quantitative and qualitative). Technical data analysis statistics were applied to the information collected concerning the total sample (495). The content analysis (qualitative approach) was applied at the open of 40 teachers from the sample answers. The results indicated that the efficacy beliefs (self-efficacy and collective efficacy) has no effect on satisfaction in teaching and other factors such as economic, may be more related to satisfaction.
\end{abstract}

Keywords: job satisfaction; teachers; collective efficacy.

\section{Resumen}

La satisfacción en el trabajo docente: Un análisis desde el modelo cognitivo social de la satisfacción en el trabajo y de la eficacia colectiva de los profesores. Mientras que la satisfacción en el trabajo es un aspecto que tiene un efecto sobre la motivación de los profesores, es necesario investigar sus implicaciones en la enseñanza. En este sentido, el objetivo de este estudio fue identificar los factores relacionados con la satisfacción en el trabajo de la evaluación de las dimensiones del modelo social de la satisfacción en el trabajo cognitivo (MSCST) y la eficacia colectiva. Por tanto, la investigación se llevó a cabo sobre una muestra de 495 profesores de una red privada de enseñanza, utilizando el enfoque mixto (cuantitativo y cualitativo). Las técnicas estadísticas de análisis de datos se aplican a la información recopilada en relación con el total de la muestra (495). El análisis de contenido (enfoque cualitativo) fue aplicado a las respuestas abiertas de 40 profesores pertenecientes a la muestra. Los resultados indicaron que las creencias de eficacia (eficacia y la eficacia colectiva) no tiene efecto sobre la satisfacción en el trabajo de enseñanza y que otros factores, tales como la económica, pueden estar más relacionados con la satisfacción.

Palabras clave: satisfacción en el trabajo; profesor; eficacia colectiva. 
O nível de satisfação com o trabalho dos profissionais em geral, e em particular dos professores com o trabalho tem papel importante na forma como as tarefas são cumpridas. Entende-se que a satisfação estimula o entusiasmo e o comprometimento, favorecendo a dedicação de mais tempo e energia à melhoria da aprendizagem dos alunos. A literatura aponta evidências que revelam a influência da satisfação na conduta profissional (Bogler \& Nir, 2012).

A satisfação no trabalho de professores tem sido relacionada ao nível de desempenho docente e à eficiência no alcance dos objetivos de ensino. Entende-se que professores mais satisfeitos com a função obtêm melhores resultados e por isso tem sido considerada como um aspecto fundamental da docência. Melhores níveis de satisfação podem resultar na melhoria dos resultados escolares. Sendo assim, considerando sua importância no contexto educacional, mais pesquisas pautadas na Teoria Social Cognitiva têm surgido nos últimos anos (Bogler \& Nir, 2012; Caprara, Barbaranelli, Steca, \& Malone, 2006). No entanto, mais estudos são necessários para aprofundar a compreensão sobre este construto e suas implicações no contexto escolar (Klassen, Usher, \& Bong, 2010).

De forma geral, este construto envolve o senso de realização profissional e pode ser influenciado por diferentes fatores emergentes da rotina de trabalho, tais como: a participação na tomada de decisão, autoestima, liberdade e independência, entre outros. A satisfação, na perspectiva social cognitiva, tem sido descrita tanto por meio de aspectos cognitivos, quanto afetivos. É, portanto, um estado emocional agradável ou positivo resultante da avaliação que o professor faz das experiências do seu trabalho (Bogler \& Nir, 2012).

A literatura baseada na Teoria Social Cognitiva (TSC) de Albert Bandura tem indicado que algumas variáveis têm efeito sobre a satisfação no trabalho docente, dentre estas se destacam as que compõem o Modelo Social Cognitivo de Satisfação no Trabalho (MSCST) (Badri, Mohaidat, Ferrandino, \& El Mourad, 2013; Duffy \& Lent, 2009; Lent et al, 2011).

Modelo social cognitivo de satisfação no trabalho (MSCST). O MSCST, Constituído originalmente por Duffy e Lent (2009), utiliza um grupo de variáveis para explicar como a satisfação do professor se desenvolve. A versão deste Modelo direcionada especificamente a professores é composta, além da variável satisfação, pelos seguintes fatores: autoeficácia, condições de trabalho, afeto positivo, progresso de objetivos e metas de apoio. Considerase que estes fatores têm efeito sobre a satisfação e, portanto, podem explicá-la (Duffy \& Lent, 2009).

A autoeficácia docente refere-se aos julgamentos que os professores fazem das suas próprias capacidades para executar as ações necessárias para atingir os objetivos propostos. Diversos estudos têm atestado sua influência sobre a satisfação no trabalho (Collie, Shapka, \& Perry, 2012; Klassen \& Chiu, 2010; Klassen, Foster, Rajani, \& Bowman, 2009; Moè, Pazzaglia, \& Ronconi, 2010; Stephanou, 2013). Moè et al. (2010)encontraram efeito direto $(p<.05 ; .32)$ da autoeficácia sobre a satisfação, o que indica que professores mais satisfeitos com o trabalho têm autoeficácia elevada.

A variável condições de trabalho dominou as pesquisas sobre satisfação no trabalho na área da psicologia comportamental e administrativa por muitos anos (Lent et al, 2011). Apesar disso, em relação a amostras de professores, poucos são os estudos que investigaram esta temática (Ramos, Costa, Pontes, Fernandez, \& Nina, 2014). No MSCST (Lent et al, 2011), esta variável é formada por duas dimensões: (1) percepção de pessoa-ambiente - que refere-se às relações e o envolvimento do indivíduo no ambiente de trabalho; (2) suporte organizacional percebido que se relaciona à percepção que o professor tem de recebimento de apoio organizacional. Duffy e Lent (2009) alertam que este construto tem recebido pouca atenção por parte dos pesquisadores desta área, sendo necessários mais estudos para investigar este aspecto.

$\mathrm{O}$ afeto positivo, outro elemento constituinte do MSCST (Duffy \& Lent, 2009), também é outra importante fonte de influência da satisfação no trabalho docente. Um ambiente com clima institucional positivo tem maiores chances de promover o sentimento de bem-estar. O afeto positivo tem ligações com outros componentes do MSCST, pois um professor que atua num ambiente de trabalho com clima institucional negativo pode ser inclinado a duvidar das suas próprias capacidades de executar tarefas (autoeficácia), influenciando também a percepção do apoio organizacional (condições de trabalho) que recebe (Lent \& Brown, 2008). 
A variável progresso de objetivos refere-se à percepção dos professores quanto aos avanços em direção ao alcance dos objetivos estipulados. Entendese que quanto mais os professores progridem nas metas idealizadas referentes ao processo de ensinoaprendizagem, maiores são as chances de sentirem-se satisfeitos com seus trabalhos. Um comportamento direcionado por metas pode ser um elemento favorável para a elevação dos níveis de satisfação, caso os objetivos estejam sendo alcançados, pois empregam um senso de propósito e direção. O sucesso no alcance dos objetivos pode ser gratificante para o professor, impulsionando seu estado motivacional (Lent \& Brown, 2008).

Metas de apoio refere-se à percepção do professor quanto ao apoio relevante recebido no ambiente de trabalho, importante para o exercício da sua função (Lent \& Brown, 2008). Lent et al (2011) identificaram, em seus estudos, que esta variável teve efeito direto $(p<.01 ; .40)$ sobre a satisfação. O MSCST de Duffy e Lent (2009) inclui alguns fatores potencialmente explicativos da satisfação, no entanto, entende-se que outros aspectos podem ser considerados, tais como a satisfação com a vida, incluída no Modelo por pesquisas posteriores a de Duffy e Lent (2009), e a eficácia coletiva, variável não pertencente ao MSCST, mas reconhecidamente relacionada à satisfação no trabalho (Caprara, Barbaranelli, Borgogni, \& Steca, 2003).

Satisfação com a vida e eficácia coletiva de professores. Em 2011, Lent et al. incluíram no MSCST a variável satisfação com a vida, considerando que este também é um importante elemento explicativo da satisfação no trabalho. Os pesquisadores afirmam que há uma conexão conceitual entre os construtos, observando a relação "parte-todo". Isto porque o trabalho do indivíduo faz parte da sua vida, e que um aspecto não pode ser investigado sem levar em consideração o outro, pois são complementares e interdependentes. Entende-se que pessoas que geralmente são felizes com suas vidas estão suscetíveis a encontrar felicidade em outros domínios específicos, tais como o trabalho. Sendo assim, o MSCST, aplicado a professores a partir de 2011, adicionou a visão de que a satisfação no trabalho e com a vida são dois aspectos que se influenciam bidirecionalmente, ou seja, de forma recíproca.

Embora não faça parte do MSCST, outro fator importante para a prática docente é a eficácia coletiva. Segundo a TSC, a eficácia coletiva de professores representa o nível de confiança que os indivíduos têm na capacidade do corpo docente, do qual fazem parte, para organizar e executar tarefas necessárias para o alcance dos objetivos idealizados. São crenças compartilhadas que refletem o quanto os professores acreditam em seus grupos. Estas crenças influenciam os aspectos normativos e culturais do ambiente de trabalho, pois têm efeito sobre a forma como os membros do grupo se envolvem na execução das tarefas. A eficácia coletiva é essencial para o funcionamento organizacional, pois as respostas dos membros do corpo docente aos problemas emergentes da rotina escolar estão fortemente relacionadas à percepção que os indivíduos têm sobre a capacidade do grupo a que pertencem, estando associada aos níveis de esforço, persistência, níveis de estresse e de desempenho, entre outros (Bandura, 1993, 1997). Desta forma, um forte senso de eficácia coletiva promove a resolução criativa de problemas e a busca dos resultados planejados.

Considerando o contexto apresentado, o presente estudo tem como objetivo: identificar fatores relacionados à satisfação no trabalho através da análise das dimensões do MSCST de Lent et al (2011) e da eficácia coletiva.

\section{Método}

\section{Tipo do Estudo}

Este estudo consiste em uma pesquisa mista (quantitativa e qualitativa), de caráter, exploratório, descritivo e inferencial para analisar as relações entre as variáveis que compõem o Modelo Social Cognitivo de Satisfação no Trabalho Docente (Lent et al., 2011) e a eficácia coletiva.

\section{Amostragem}

Procedeu-se o cálculo amostral visando generalizar os resultados obtidos na população alvo. Neste estudo, aplicou-se amostragem aleatória simples numa população de 1079 professores dos estados do Pará, Amapá e Maranhão, que atuam numa rede de escolas confessionais, nos tipos de Educação Infantil, Ensino Fundamental e Médio. Sendo assim alcançou-se uma amostra de 495 docentes, com um erro amostral de $3,31 \%$.

\section{Instrumentos de Coleta}

Utilizaram-se três instrumentos para coleta dos dados: o Questionário de Caracterização do Participante; 
a escala de Crença Coletiva Escolar - com o objetivo de medir as crenças dos professores acerca da capacidade coletiva para influenciar o desempenho acadêmico dos alunos; e o questionário Pensamentos sobre Ensino $e$ Escola (Lent et al., 2011) - que avalia as sete variáveis que compõem o Modelo Social Cognitivo de Satisfação no Trabalho e suas relações, a saber: apoio de eficácia relevante, condições de trabalho (apoio organizacional percebido), progresso de objetivos, satisfação com a vida, satisfação com o trabalho, afeto positivo e autoeficácia. De forma total, os participantes responderam a 107 itens, na somatória de todas as escalas e questionários.

\section{Procedimentos de Coleta}

$\mathrm{Na}$ aplicação dos questionários os participantes receberam informações referentes à pesquisa, esclarecimentos sobre a função do Termo de Consentimento Livre Esclarecido (TCLE) e orientações para preenchimento dos instrumentos. A coleta foi realizada de forma coletiva, pois todos os instrumentos são auto aplicativos. No entanto, todo o processo foi acompanhado pela pesquisadora que apresentou novas orientações e esclarecimentos, quando necessário.

\section{Procedimento de Análise}

Análises quantitativas e técnicas estatísticas. $\mathrm{Na}$ fase das análises quantitativas, utilizaram-se três técnicas estatísticas para a investigação das respostas dos 495 professores participantes da presente pesquisa, sendo estas: (a) Análise Fatorial (AF); (b) Análise de Correlação de Pearson (r); (c) Regressão Linear Simples. Por meio da análise fatorial, construíram-se índices (fatores) referentes às oito variáveis investigadas quantitativamente neste estudo, a saber: as sete dimensões do MCSST (satisfação no trabalho, satisfação com a vida, progresso de objetivos, apoio de eficácia relevante, condições de trabalho, afeto positivo, autoeficácia) e a eficácia coletiva.

Cumpriram-se as seguintes etapas para a construção dos índices de cada variável envolvida na pesquisa, a saber: (a) teste de normalidade e identificação da existência ou não de outliers (valores discrepantes no conjunto de dados); (b) análise da matriz de correlação - considera-se que as correlações devem ter valores iguais ou maiores que 0,30 , neste estudo todos os valores foram superiores a 0,30; (c) estatística de Kaiser-Meyer-Olkin (KMO) - para indicar se existe uma adequação à análise fatorial, com valores maiores que 0,8; (d) teste de esfericidade de Bartlette; (e) Medida de Adequação da Amostra - superiores à 0,7 (MAA) - para verificar se as variáveis em estudo são apropriadas à utilização da técnica AF; (f) critério de Kaiser para determinar a quantidade de fatores extraídos, isto é, quantidade de equações necessárias a construção dos índices; (g) método Varimax - para rotacionar os valores extraídos para que cada fator possa maximizar a informação de cada variável utilizada na construção dos índices; (h) cálculo de escores fatoriais (índices) de cada professor a partir da multiplicação dos valores individuais atribuídos a cada pergunta por professor pelos pesos fatoriais, (i) padronização dos valores obtidos, para que os mesmos pudessem ser avaliados em uma escala de 0 a 1 ou 0 a $100 \%$. Ressalta-se que em todas essas fases as variáveis em investigação se demonstram adequadas para a análise fatorial, sendo possível calcular todos os índices.

Após obtenção dos índices de cada variável, aplicou-se a análise de correlação de Pearson $(r)$ para verificar a existência de relacionamento, servindo como base para estimar todas as relações da Regressão Linear Simples.

Posteriormente, realizou-se a análise de Regressão Linear Simples para estimar os valores da variável de critério, com base nos valores conhecidos ou fixados de cada variável explicativa. Aplicou-se esta técnica apenas em casos de correlações significativas. Considerou-se a seguinte estrutura para investigar empiricamente as associações entre as dimensões teóricas: variável de critério - satisfação no trabalho; variáveis explicativas (analisadas separadamente) - autoeficácia, condições de trabalho, progresso de objetivos, apoio de eficácia relevante, afeto positivo, satisfação com a vida, eficácia coletiva (Corrar, Paulo, \& Dias Filho, 2007).

Analisou-se ainda a relação explicativa entre a eficácia coletiva e cada componente do MSCST, com exceção da satisfação no trabalho, para verificar se as crenças coletivas têm algum efeito sobre os fatores potencialmente preditores da satisfação a partir do MSCST de Lent et al. (2011). Para a aplicação das técnicas estatísticas desta fase de análises quantitativas, utilizouse o pacote estatístico SPSS - 20.

Análise qualitativa - Análise de conteúdo. Para as análises relativas à questão aberta disposta no instrumento de coleta, consideraram-se as informações (relatos) disponibilizadas por 40 professores mediante uma questão aberta presente no grupo de instrumentos de coleta. Por ser de caráter opcional, apenas $8 \%$ 
dos docentes envolvidos na pesquisa decidiram contribuir com suas respostas nesta questão. O referido item possibilitou aos participantes acrescentarem informações (adicionais) que considerassem importantes e que julgassem não ter sido abordadas ou devidamente evidenciadas nos instrumentos utilizados. Utilizou-se análise qualitativa entendendo que os relatos estão em formato textual, sendo necessário, assim, investigar as categorias emergentes dos trechos textuais.

Para tanto, aplicou-se a análise de conteúdo por meio do software NVIVO 10. Essa ferramenta foi desenvolvida, visando promover uma melhor organização, análise e visualização dos dados. Nesta etapa, objetivou-se identificar outros fatores associados à satisfação no trabalho docente.

As respostas dos participantes foram inseridas no software NVIVO 10 para tratamento dos dados. Procedeu-se à codificação, que consiste em reunir informações por tópicos, temas ou casos, criando "nós" (categorias). Assim, criou-se categorias a partir das fontes de informações (respostas dos participantes) inseridas no software. Ressalta-se que para a descrição das respostas dos professores foram utilizados nomes fictícios, para proteger suas identidades.

Enfatiza-se que o foco desta etapa do estudo foi analisar os principais fatores que emergiram dos relatos dos participantes. A descrição dos dados seguiu a ordem de importância, dada a partir da frequência de incidência.

\section{Resultados das análises quantitativas}

\section{Estudo das Relações Explicativas}

Inicialmente verificou-se as correlações significativas entre as dimensões do MSCST e destas com a eficácia coletiva (Tabela 1) como pré-requisito para a posterior análise de Regressão Linear Simples.

A análise de correlação de Pearson indica uma medida de relação entre as variáveis. Considerou-se como significativas, ou seja, com existência de associação entre os elementos, a configuração de $p$ entre 0,00 e 0,05 (Dancey \& Reidy, 2006). Neste sentido, notou-se que a autoeficácia foi a única variável do MSCST que não se correlacionou significativamente à satisfação no trabalho docente $(p>0,05)$. Adicionalmente, a eficácia coletiva não foi relacionada à satisfação, no entanto associou-se a cinco elementos do MSCST, sendo estes: autoeficácia ( $p$ $<0,01 ; r=0,55)$; afeto positivo $(p<0,01 ; r=0,16)$; apoio de eficácia relevante $(p<0,01 ; r=0,17)$; progresso de objetivos ( $p<0,01 ; r=0,14)$; satisfação com a vida ( $p<$ 0,$01 ; r=0,12$ ).

Analisou-se a intensidade das relações entre as variáveis. Para tanto, considerou-se a seguinte regra: quanto mais próximo de 1 ou -1 for o coeficiente ( $r$ ), mais forte será a associação. Para a categorização da intensidade das relações atendeu-se os seguintes parâmetros correlacionais: sem correlação (0); fraco (0,1/-0,1 a 0,3/-0,3); moderado (0,4/-0,4 a 0,6/-0,6); forte (0,7/-0,7 a 0,9/-0,9); perfeito (1) (Dancey \& Reidy, 2006). Notou-se que todas as associações entre as variáveis que apresentaram correlações significativas dentro do MSCS foram fracas. Houve apenas uma relação moderada entre a eficácia coletiva e a autoeficácia ( $p<0,00 ; r=0,55)$, sendo que a eficácia coletiva não faz parte do Modelo e não se associou à satisfação no trabalho.

Com estes parâmetros delimitados realizouse análise de Regressão Linear Simples para avaliar as relações explicativas entre as variáveis que obtiveram correlações significativas. Incialmente, avaliou-se apenas as dimensões que compõem o MSCST (Figura 1).

Buscou-se por meio da análise de Regressão Linear Simples identificar quais variáveis propostas pelo MSCST têm a capacidade de explicar a satisfação no trabalho docente, a partir dos dados levantados nessa pesquisa. As variáveis que apresentaram correlação significativa com a satisfação também demonstraram ter efeito explicativo sobre ela, a saber: apoio de eficácia relevante $(p<0,01 ; \beta$ $=.03$ ); afeto positivo ( $p<0,01 ; \beta=.03)$; condições de trabalho $(p<0,05 ; \beta=.04)$; satisfação com a vida $(p<0,01 ; \beta=.02)$; progresso de objetivos $(p<0,05 ; \beta=.03)$.

Para avaliar a intensidade dos efeitos destas variáveis sobre a satisfação, tomaram-se por base os mesmos parâmetros aplicados ao delineamento correlacional, quanto mais próximo de $1 /-1$ for 0 coeficiente, mais forte é a capacidade de explicação. Neste sentido, observando que os coeficientes padronizados $(\beta)$ das variáveis explicativas foram fracos $(<0,1)$, calculouse o $R$ Square $\left(R^{2}\right)$, que é o coeficiente de determinação ou poder explicativo da regressão. $O \mathrm{R}^{2}$ indica quanto da variação de uma variável depedente de $y$, neste caso a satisfação no trabalho, é explicada pelas variações na variável ou conjunto de variáveis independentes ( $x$ ou $x 1$, $x 2, x 3$...). No caso deste estudo, a variação neste grupo de variáveis, pertencentes ao MSCST, explicou apenas $12 \%$ da variação da satisfação no trabalho docente, sendo que não foi possível incluir uma dimensão deste Modelo (autoeficácia), pois não houve correlação significativa com a satisfação. 
Satisfação no trabalho e eficácia coletiva docente

Tabela 1. Relacionamento entre as Variáveis.

\begin{tabular}{|c|c|c|c|c|c|c|c|}
\hline & Eficácia Coletiva & Autoeficácia & Afeto Positivo & $\begin{array}{l}\text { Apoio de eficácia } \\
\text { relevante }\end{array}$ & $\begin{array}{l}\text { Condições de } \\
\text { trabalho }\end{array}$ & $\begin{array}{c}\text { Progresso de } \\
\text { objetivos }\end{array}$ & $\begin{array}{c}\text { Satisfação com } \\
\text { a Vida }\end{array}$ \\
\hline Autoeficácia & $0,557^{*}$ & & & & & & \\
\hline Afeto Positivo & $0,161^{*}$ & $0,189^{*}$ & & & & & \\
\hline $\begin{array}{l}\text { Apoio de eficácia } \\
\text { relevante }\end{array}$ & $0,175^{*}$ & $0,158^{*}$ & $0,241^{*}$ & & & & \\
\hline $\begin{array}{l}\text { Condições de } \\
\text { trabalho }\end{array}$ & 0,002 & $-0,011$ & 0,070 & 0,079 & & & \\
\hline $\begin{array}{l}\text { Progresso de } \\
\text { objetivos }\end{array}$ & $0,143^{*}$ & $0,180^{*}$ & $0,208^{*}$ & $0,265^{*}$ & $0,038^{* *}$ & & \\
\hline $\begin{array}{l}\text { Satisfação com } \\
\text { a Vida }\end{array}$ & $0,129^{*}$ & $0,172^{*}$ & $0,308^{*}$ & $0,256^{*}$ & 0,066 & $0,150^{*}$ & \\
\hline $\begin{array}{l}\text { Satisfação no } \\
\text { Trabalho }\end{array}$ & 0,000 & 0,038 & $0,162^{*}$ & $0,172^{*}$ & $0,156^{*}$ & $0,122^{*}$ & $0,304^{*}$ \\
\hline
\end{tabular}

Nota: ${ }^{*} p<0.01 ;{ }^{* *} p<0.05$.

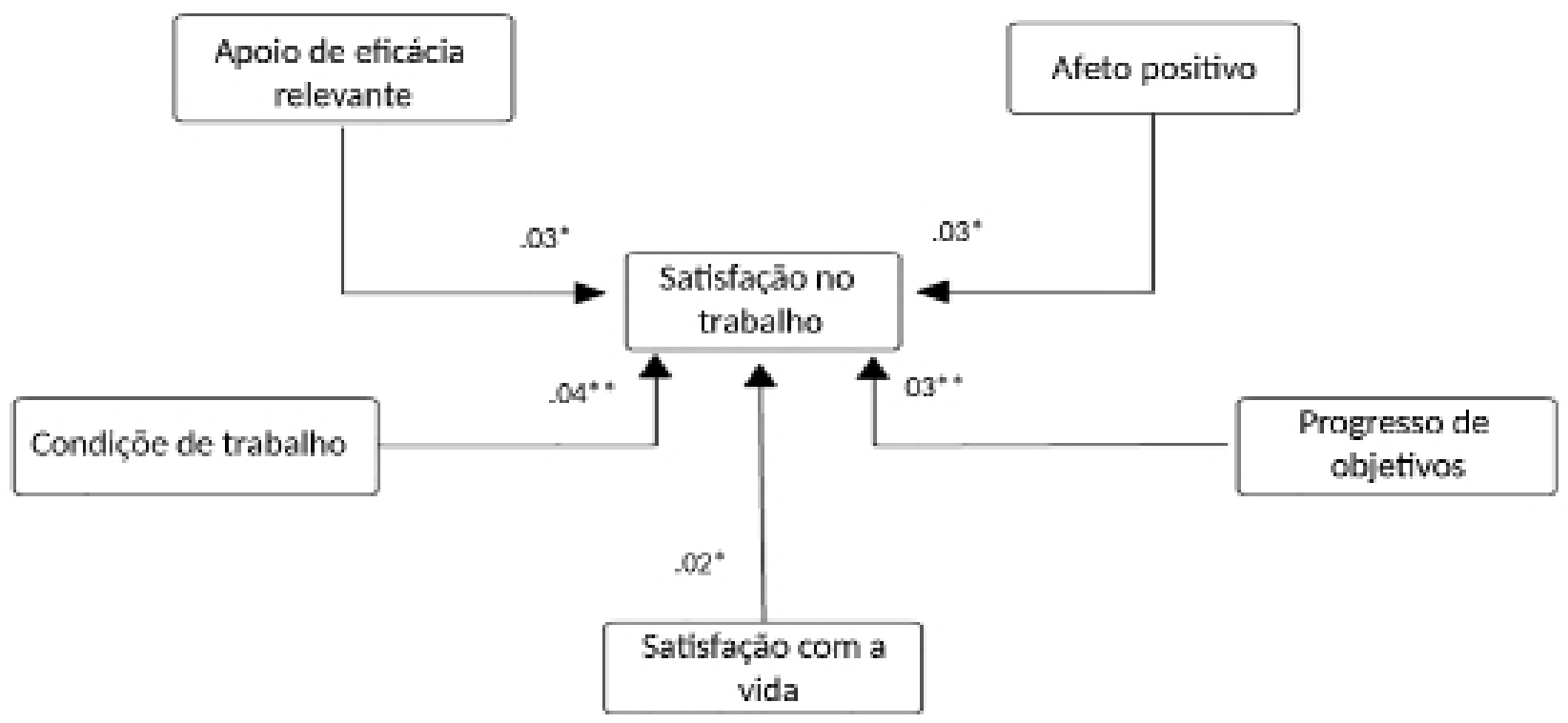

Figura 1. Relações Explicativas entre as Variáveis do MSCST. ${ }^{*} p<0.01 ;{ }^{* *} p<0.05$.

Isoladamente, as variáveis apoio de eficácia relevante, afeto positivo, condições de trabalho, progresso de objetivos e satisfação com a vida também apresentaram pouca capacidade de explicação referentes à satisfação no trabalho docente ( $<1 \%$ cada uma). Isto sugere que tanto isoladamente, quanto em conjunto as variáveis do MSCST explicam pouco a variação dos níveis de satisfação dos professores que compõem a amostra selecionada.

Relações explicativas entre a eficácia coletiva docente e as variáveis do MSCST. Apesar de não ter havido correlação significativa entre a eficácia coletiva e duas dimensões do MSCST (satisfação no trabalho e condições de trabalho), verificou-se as relações explicativas entre as crenças coletivas e as demais variáveis do MSCST, com as quais se associou significativamente, buscando identificar se a eficácia coletiva tem efeito sobre fatores que influenciam a satisfação (Figura 2).

Notou-se que a eficácia coletiva explicou, de forma fraca, cinco dimensões do MSCST, a saber: satisfação com a vida $(p<0,05 ; \beta=.06)$; progresso de objetivos ( $p<0,05 ; \beta=.03)$; apoio de eficácia relevante ( $p<0,01 ; \beta=.05)$; afeto positivo $(p<0,01 ; \beta=.05)$; autoeficácia $(p<0,01 ; \beta=.03)$. 


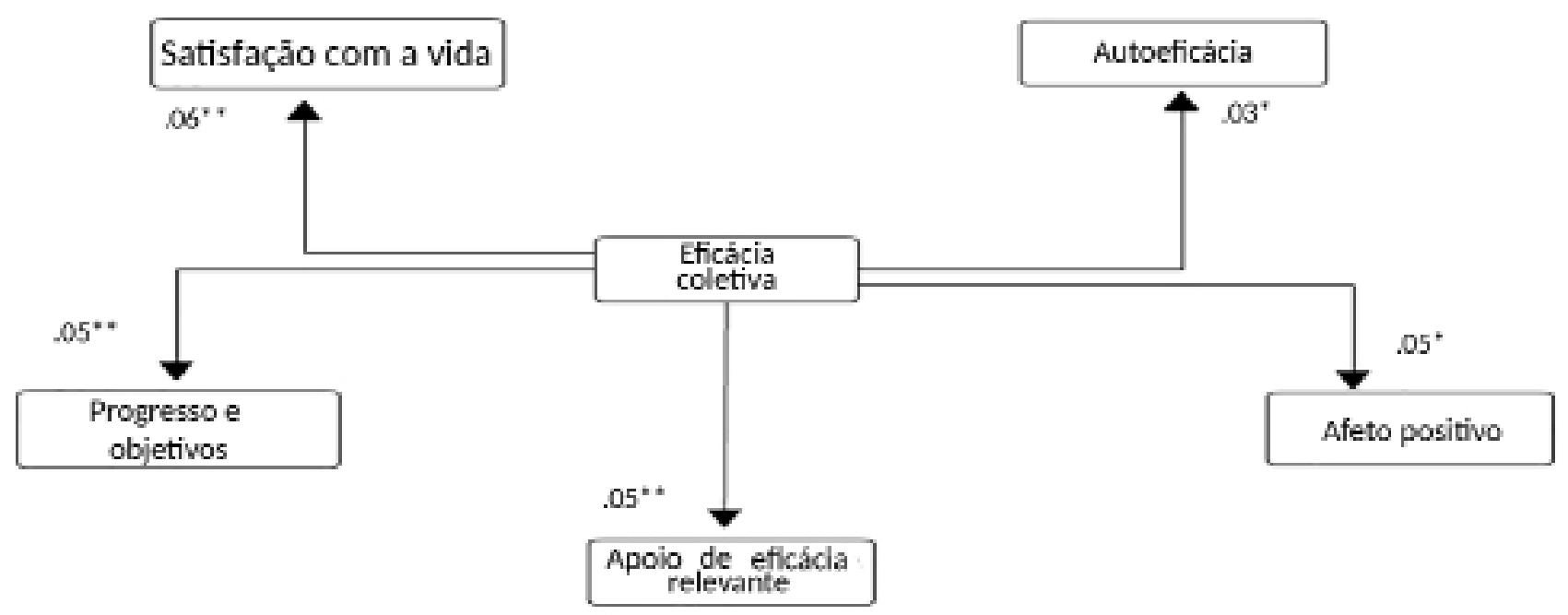

Figura 2. Relações Explicativas entre a Eficácia Coletiva e as Dimensões do MSCST, com Exceção das Variáveis Satisfação e Condições de Trabalho. * $p<0.01$; ** $p<0.05$.

\section{Resultados da análise qualitativa}

As respostas à questão aberta foram organizadas por meio de análise de conteúdo. Os 40 professores participantes desta etapa tiveram a oportunidade de registrar informações adicionais relacionadas à satisfação no trabalho docente referente às práticas docentes em contexto escolar. A questão foi a seguinte: "Caso queira, utilize o espaço abaixo para realizar algum comentário relacionado aos aspectos contidos nos questionários que respondeu (sobre satisfação no trabalho). Você também pode comentar algo que não foi contemplado nos questionários, mas que você considera importante." A Figura 3 apresenta a estrutura de codificação realizada por meio do software NVIVO 10 para a identificação de fatores relacionados à satisfação no trabalho, indicados nos relatos dos participantes.

A Figura 3 demonstra a forma como as fontes de dados foram codificadas. As categorias criadas emergiram das informações disponibilizadas pelos participantes e foram pautadas na Teoria Social Cognitiva. Ressalta-se que a soma da frequência de ocorrência das categorias na Figura 3 é superior ao total de participantes (40), pois não são exclusivas. Assim, é possível visualizar as categorias e a frequência de relatos referentes a cada uma delas. Desta forma, observou-se que os aspectos mais evidenciados foram: insatisfação com o salário (17 relatos); estados emocionais (oito relatos); relação professor-aluno (quatro relatos); suporte e apoio organizacional (quatro relatos).
Outros aspectos, no entanto, foram mencionados com frequência igual ou menor que três, a saber: sobrecarga de trabalho (três relatos); pressão do tempo (dois relatos); valorização e reconhecimento (dois relatos); quantidade de alunos por sala (dois relatos); relação com os colegas de trabalho (dois relatos); indisciplina escolar (dois relatos); insatisfação com a vida (um relato); infraestrutura da escola e adoecimento docente (um relato).

Insatisfação com o salário. A categoria insatisfação com o salário foi a mais mencionada pelos participantes, estando presente em $43 \%$ dos relatos. As respostas de 17 professores sobre a insatisfação salarial evidenciaram que os participantes entendem que é prazeroso o exercício da docência, no entanto a recompensa (salário) é considerada desanimadora e, por vezes, os docentes precisam trabalhar com carga horária maior (dupla ou tripla jornada), ou mesmo realizar outras atividades para complementar o salário. Sobre isso a professora Ana destacou:

Muitas vezes me sinto desanimada, não disposta para dar as aulas por que a escola não nos incentiva, não somos valorizados, e o salário desanima qualquer um. Tento dar o melhor, mas quando não há apoio fica difícil. Eu gosto do que eu faço, embora tenha que fazer outras atividades para melhorar o meu salário, pois infelizmente não dá pra suprir minhas necessidades (Professora Ana). 


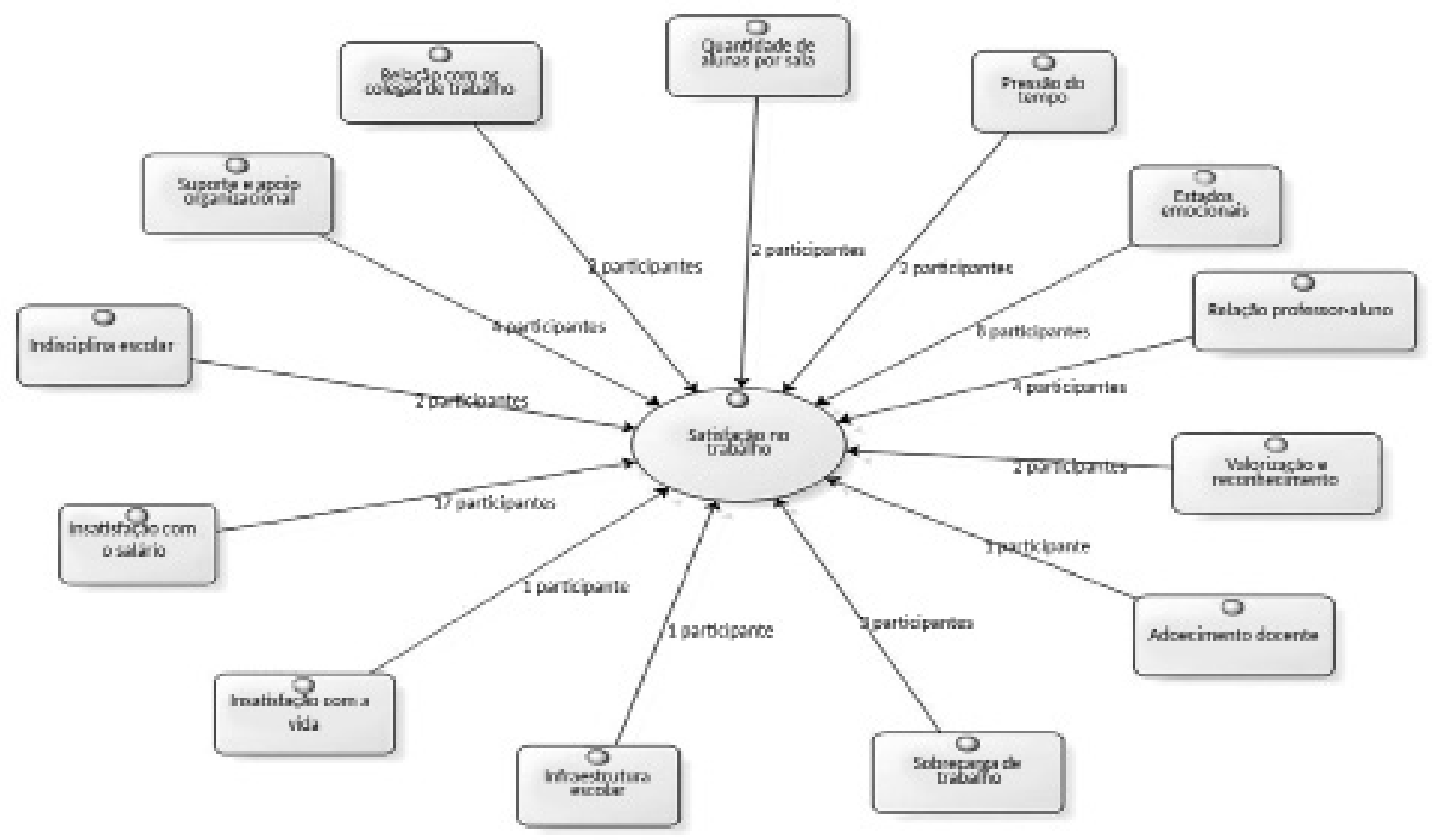

Figura 3. Codificação das Fontes de Informações.

Percebeu-se, ainda, que os professores associam a questão salarial à sobrecarga de trabalho, ao adoecimento docente e à diminuição da satisfação no trabalho. Os relatos indicam que os docentes entendem que seus salários não estão de acordo com a complexidade da função que exercem, em alguns casos, com o seu nível de formação. Consideram isso um fator de desvalorização que não é apenas local (realidade da escola em que trabalham), mas é um problema em nível de políticas educacionais nacionais de recompensa.

$\mathrm{Em}$ alguns casos os docentes manifestaram que o salário que recebem não é suficiente para atender as suas necessidades, como enfatizado pela professora Ana, questões como essas atingem o estado motivacional dos professores, causando desânimo e consequentemente diminuindo a satisfação.

Estados emocionais. A categoria estados emocionais esteve presente em $20 \%$ das respostas (oito incidências). Notou-se que nos relatos obtidos quatro participantes indicaram que tentam se manter motivados e com bom ânimo, apesar das muitas dificuldades relacionadas ao contexto escolar, tais como os problemas de relacionamento com pais, alunos, colegas e superiores e falta de apoio institucional. Defendem que o estado emocional positivo contribui para o melhor cumprimento das tarefas e para a permanência na docência.
No entanto, outros quatro participantes destacaram que em virtude das dificuldades inerentes à profissão, seus estados motivacionais foram abalados e não encontram ânimo para realizar satisfatoriamente as atividades referentes à rotina escolar. A professora Maria ressaltou:

Tem dias que amanhece chovendo, nestes dias eu não desejo nem levantar da cama, estou cansada! Há dias em que os pais fazem críticas duras, nestes dias eu me sinto injustiçada. Sinto-me muito desanimada! (Professora Maria).

Notou-se a existência de professores resilientes e outros que demonstram uma postura mais reativa frente aos problemas da docência, especialmente referentes aos relacionamentos com pais, alunos, colegas de trabalho e superiores. Pelos relatos, os participantes que informaram estar desanimados e estressados, também revelaram estar insatisfeitos com seus trabalhos.

Relação professor-aluno. A categoria relação professor-aluno foi mencionada por quatro participantes (15\%). Em três casos os professores informaram que encontram nos relacionamentos com os alunos o incentivo necessário para realizar as tarefas da docência da melhor forma possível e que este é um importante 
aprendizado para eles, que se dá por meio das trocas de experiências com os alunos. Neste sentido a relação com os discentes foi mais positivamente destacada pelos participantes. O professor João enfatizou:

Eu me sinto interessado, animado, entusiasmado, dentre outros atributos, quando estou dentro da sala de aula, no convívio com os alunos, no processo ensino-aprendizagem, é simplesmente gratificante (Professor João).

O fator relação professor-aluno foi um aspecto importante, sendo considerado pelos participantes como um dos principais motivos para não desistirem da profissão. Apenas um professor enfatizou o aspecto negativo nesta relação, destacando que o desinteresse dos alunos na realização das suas atividades escolares e os conflitos resultantes disso, agem como aspectos desmotivadores.

Suporte e apoio organizacional. A categoria suporte e apoio organizacional também foi mencionada em $15 \%$ das respostas dos participantes (quatro incidências). De acordo com os relatos, quando os professores recebem suporte organizacional sentem-se mais motivados para exercer a docência, no entanto quando não recebem o apoio necessário para exercer a função, sentem-se estressados e sobrecarregados. Dois participantes indicaram que o suporte que precisam está direcionado para os materiais pedagógicos e ferramentas necessárias para a prática docente, dentre estas estão as novas tecnologias de ensino. O professor José destacou:

"As escolas precisam dar mais suporte ao professor e investir em ferramentas de trabalho para sala de aula. Uma rede de escolas como esta não pode ficar atrasada em relação às tecnologias" (Professor José).

Os professores entendem que a cobrança dos pais e da comunidade escolar por uma educação de qualidade, exige maior investimento em materiais e ferramentais que ofereçam melhores condições de ensino, visando um processo de aprendizagem de qualidade. Um participante, no entanto, caracterizou o apoio que recebe no ambiente de trabalho, como um clima harmonioso, cooperativo e empático e que estes aspectos são fundamentais para que o professor sinta-se bem e realizado com seu trabalho.

\section{Discussão}

Os resultados deste estudo apresentam informações importantes sobre o MSCST, na versão de Lent et al. (2011), aplicado aos professores. Na amostra selecionada no presente estudo, a composição de variáveis do MSCST de Lent et al., (2011) se demonstrou pouco explicativa, inclusive uma das dimensões, a autoeficácia, não se relacionou à variável principal do MSCST (satisfação). Resultado semelhante foi encontrado em outros estudos que adotaram o modelo de Lent et al. (2011). Por outro lado, Duffy e Lent (2009) encontraram relação explicativa entre estas variáveis. Estes dados, no entanto, contradizem pesquisas que investigaram estes fatores fora do MSCST, indicando que a autoeficácia tem forte efeito sobre a satisfação (Bogler \& Nir, 2012; Klassen \& Chiu, 2010; Klassen, Bong, et al., 2009; Klassen, Foster, et al., 2009; Moè et al., 2010; Stephanou, 2013)teaching efficacy, and job satisfaction \u2014and to examine the interrelationships among the three outcome variables. Along with sense of job satisfaction and teaching efficacy, two types of stress (workload and student behavior stress. Ao que parece, quando estas variáveis são analisadas em conjunto, a partir do Modelo, a autoeficácia não se confirma como fator explicativo da satisfação.

Outro achado importante diz respeito à relação entre a eficácia coletiva docente e a satisfação. Neste estudo não houve qualquer associação entre estas variáveis, sendo este um resultado que contrariou pesquisas anteriores. Estudos (Caprara et al., 2003; Göker, 2012; Klassen, 2010; Klassen, Foster, et al., 2009; Stephanou, 2013)têm apontado que as crenças coletivas estão diretamente relacionadas à satisfação.

Considerando os resultados encontrados, notou-se que as crenças de eficácia (autoeficácia e eficácia coletiva) não possuem capacidade de explicar a satisfação no trabalho docente a partir da composição do MSCST, na amostra deste estudo. Apesar disso as crenças coletivas foram capazes de explicar outras variáveis que compõem o Modelo, a saber: autoeficácia, satisfação com a vida, progresso de objetivos, afeto positivo e apoio de eficácia relevante. Neste sentido, com exceção da variável autoeficácia que não apresentou qualquer relação com a satisfação, a eficácia coletiva foi capaz de explicar, mesmo que de forma fraca, fatores que têm efeito direto sobre a satisfação. Sugere-se, a partir disso, que a eficácia coletiva pode influenciar a satisfação no trabalho de forma indireta, sendo mediada pelas cinco dimensões do MSCST.

Neste estudo, o MSCST de Lent et al.(2011) não se apresentou adequado para investigar a satisfação no trabalho, pois sua capacidade de explicação foi baixa, talvez esse resultado esteja relacionado à população 
de professores de escolas religiosas, sendo assim, é importante que mais pesquisas sejam feitas envolvendo populações diferentes. Considerando os escores por dimensão do Modelo, cada variável, com correlações significativas, apresentou capacidade de explicação $<1 \%$, e o conjunto destas dimensões apenas $12 \%$. Em estudos anteriores o MSCST alcançou as seguintes capacidades de explicação: Duffy e Lent (2009) - 75\% da variância; Lent et al. (2011) - 41\%; Badri et al. (2013) - 82\%; BuyukgozeKavas et al. (2013) - 33\%. Considera-se que o Modelo não sofreu grande variação nestas pesquisas. No estudo de Lent et al.(2011) incluiu-se uma variável (satisfação com a vida) e ajustou-se o termo metas de apoio para apoio de eficácia relevante, já na pesquisa de Buyukgoze-Kavas et al.(2013) a variável metas de apoio foi suprimida.

De forma geral os resultados da análise quantitativa indicam que as variáveis que compõem o MSCST parecem não explicar suficientemente a satisfação no trabalho docente, o que se contrapõe às hipóteses testadas neste estudo. Considera-se que tais resultados podem ser explicados pela diferença de contexto, pois o MSCST foi construído inicialmente para ser aplicado em amostras de professores de escolas dos Estados Unidos (Duffy \& Lent, 2009) e docentes da Itália (Lent et al., 2011). Entende-se que fatores que se demostraram importantes nestas amostras não foram significativos em contexto brasileiro, indicando que outros elementos podem explicar melhor a satisfação no trabalho, ou mesmo justificar uma possível insatisfação profissional. Problemas relacionados à precarização do trabalho docente devem ser considerados, condições inapropriadas para o ensino, sobrecarga de trabalho, indisciplina, ou mesmo falta de suporte, entre outros, são aspectos presentes em escolas brasileiras mesmo pertencentes às redes privadas de ensino (Ramos, 2015). Estes fatores foram identificados nos relatos dos participantes desta pesquisa na fase qualitativa deste estudo. Considera-se, ainda, importante identificar variáveis para a construção de um Modelo de Satisfação no Trabalho Docente que de fato contemple as características do contexto brasileiro, sendo necessários mais estudos para identificar e testar estas possíveis dimensões.

A análise qualitativa, pautada nas respostas abertas de 40 professores da amostra total selecionada (495), relevou que outros elementos emergentes da rotina de trabalho podem estar associados à satisfação. Estes professores indicaram um grupo de fatores que não foram considerados no MSCST, sendo estes: insatisfação com o salário; relação professor-aluno; sobrecarga de trabalho; pressão do tempo; valorização e reconhecimento; quantidade de alunos por sala; relação com os colegas de trabalho; indisciplina escolar; insatisfação com a vida; infraestrutura da escola e adoecimento docente.

Houve destaque para a insatisfação com o salário que esteve presente em $43 \%$ dos relatos, este é um fator extrínseco que tem sido relacionado à satisfação no trabalho (Bogler \& Nir, 2012; Canrinus, Helms-Lorenz, Beijaard, Buitink, \& Hofman, 2011). Notou-se que este aspecto foi associado pelos participantes da pesquisa ao sentimento de desvalorização da profissão e de falta de melhores políticas públicas de remuneração docente. Enfatiza-se que o MSCST de Lent et al., (2011) não contemplou esta variável. Considera-se que isto ocorreu pois esta é uma problemática emergente tipicamente do contexto brasileiro, onde a remuneração do professor é um dos aspectos canalizadores do sentimento de desvalorização do docente.

Alves e Pinto (2011) indicam que um professor bem remunerado e com perspectiva de crescimento pode apresentar melhor desempenho, no entanto em situações profissionais precárias não há estímulo para uma boa atuação. Reconhece-se que professores, em busca de melhores padrões de remuneração, têm ampliado sua jornada de trabalho e, em alguns casos, isto afeta negativamente o exercício da profissão (Pinto \& Alves, 2011).

Como indicado pelos participantes desta pesquisa, a insatisfação com o salário é um problema em nível de políticas nacionais de educação. Segundo dados da Organização para a Cooperação e Desenvolvimento Econômico (2014) (OCDE) os salários dos professores brasileiros estão entre os mais baixos dos países desenvolvidos. Mesmo considerando países da América Latina, como o Chile e México a remuneração do professor brasileiro é consideravelmente mais baixa. $\mathrm{O}$ Brasil fica a frente apenas da Indonésia, no ranking dos países desenvolvidos. Esta pesquisa toma por base os dados de 2012.

Segundo o último relatório do Conselho de Desenvolvimento Econômico e Social (2014), o rendimento médio do professor da Educação Básica, em 2012, foi equivalente a $51,7 \%$ do rendimento médio obtido pelos demais profissionais com nível superior. Sendo assim a questão salarial é, portanto, um fator que deve ser considerado ao se analisar o nível de satisfação do professor brasileiro com sua função. 
Bogler e Nir (2012), em seus estudos, sugeriram que a insatisfação com o salário tem capacidade de influenciar negativamente e fortemente a satisfação profissional do professor e que, inclusive, impulsiona o desejo de desistir da função. Göker (2012) indicou que professores geralmente sentem-se realizados com alguns aspectos da docência mais relacionados com o processo de ensino e o crescimento profissional, no entanto, estão insatisfeitos com aspectos que envolvem as condições de trabalho e recompensa (salário).

Notou-se, ainda, que os relatos obtidos a partir da questão aberta, aplicada aos 40 participantes indicaram dois aspectos, os quais foram associados à satisfação no trabalho e considerados no MSCST de Lent et al. (2011), a saber: suporte e apoio organizacional, que no Modelo é representado pelo termo condições de trabalho; e estados emocionais, tidos no MSCST como afeto positivo. Ambas as dimensões apresentaram relações explicativas significativas, embora fracas, com a satisfação no trabalho a partir das análises quantitativas aplicadas ao total de participantes desta pesquisa (495). Sendo assim estes resultados quantitativos relacionados ao Modelo corroboram com a emergência destas categorias no relato dos participantes.

\section{Considerações finais}

Os resultados deste estudo podem servir como base para repensar políticas internas, visando aprimorar práticas institucionais para tornar o professor mais satisfeito com seu trabalho. As principais contribuições desta pesquisa foram a testagem do MSCTS em amostras brasileiras, a testagem da inclusão da variável eficácia coletiva ao Modelo e o levantamento de outros possíveis elementos explicativos da satisfação no trabalho docente mais emergentes de escolas do Brasil, sendo especificamente escolas privadas e confessionais.

Considera-se importante que instrumentos de coleta construídos em contextos socioculturais distintos passem por uma adaptação contextual para garantir a validade do instrumento no Brasil.

Sugere-se que outros estudos sejam realizados para investigar a relação entre a eficácia coletiva e a satisfação, envolvendo, inclusive a aplicação de outros instrumentos apontados na literatura da psicologia social cognitiva. Tendo em vista que este estudo investigou professores de uma rede privada de escolas confessionais, sendo esta uma limitação desta pesquisa, sugere-se que outros estudos sejam realizados em amostras de professores da rede pública de ensino.

\section{Referências}

Alves, T., \& Pinto, J. M. R. (2011). Remuneração e características do trabalho docente no Brasil: um aporte dos dados do Censo Escolar e da PNAD. Cadernos de Pesquisa, 41(143), 606-639. doi: 10.1590/ S0100-15742011000200014

Badri, M. A., Mohaidat, J., Ferrandino, V., \& El Mourad, T. (2013). The social cognitive model of job satisfaction among teachers: Testing and validation. International Journal of Educational Research, 57, 12-24. doi: 10.1016/j.ijer.2012.10.007

Bandura, A. (1993). Perceived self efficacy in cognitive development and functioning. Educational Psychologist, 28(2), 117-148. doi: 10.1207/ s15326985ep2802_3

Bandura, A. (1997). Self-efficacy: The exercise of control. Recuperado de http://books.google.com.br/books/about/Self_Efficacy.html?id=eJ-PN9g_o-EC\&pgis=1

Bogler, R., \& Nir, A. E. (2012). The importance of teachers' perceived organizational support to job satisfaction: What's empowerment got to do with it? Journal of Educational Administration, 50(3), 287-306. doi: 10.1108/09578231211223310

Buyukgoze-Kavas, A., Duffy, R. D., Guneri, O. Y., \& Autin, K. L. (2013). Job satisfaction among turkish teachers: Exploring differences by school level. Journal of Career Assessment, 22(2), 261-273. doi: $10.1177 / 1069072713493980$

Canrinus, E. T., Helms-Lorenz, M., Beijaard, D., Buitink, J., \& Hofman, A. (2011). Self-efficacy, job satisfaction, motivation and commitment: Exploring the relationships between indicators of teachers' professional identity. European Journal of Psychology of Education, 27(1), 115-132. doi: 10.1007/s10212-011-0069-2

Caprara, G. V., Barbaranelli, C., Borgogni, L., \& Steca, P. (2003). Efficacy beliefs as determinants of teachers' job satisfaction. Journal of Educational Psychology, 95(4), 821-832. doi: 10.1037/00220663.95.4.821

Caprara, G. V., Barbaranelli, C., Steca, P., \& Malone, P. S. (2006). Teachers' self-efficacy beliefs as determinants of job satisfaction and students' academic achievement: A study at the school level. Journal of School Psychology, 44(6), 473-490. doi: 10.1016/j.jsp.2006.09.001

Collie, R. J., Shapka, J. D., \& Perry, N. E. (2012). School climate and social-emotional learning: Predicting teacher stress, job satisfaction, and teaching efficacy. Journal of Educational Psychology, 104(4), 1189-1204. doi:10.1037/a029356

Corrar, L. J., Paulo, E., \& Dias Filho, J. M. (2007). Análise multivariada: para os cursos de administração, ciências contábeis e economia. FIPECAFI. São Paulo: Atlas.

Dancey, C. P., \& Reidy, J. (2006). Estatística sem matemática para psicologia. Porto Alegre: Artmed.

Duffy, R. D., \& Lent, R. W. (2009). Test of a social cognitive model of work satisfaction in teachers. Journal of Vocational Behavior, 75(2), 212-223. doi: 10.1016/j.jvb.2009.06.001

Göker, S. D. (2012). Impact of EFL teachers‘ collective efficacy and job stress on job satisfaction. Theory and Practice in Language Studies, 2(8), 1545-1551. doi: 10.4304/tpls.2.8.1545-1551 
Klassen, R. M. (2010). Teacher stress: The mediating role of collective efficacy beliefs. The Journal of Educational Research, 103(5), 342350. doi: 10.1080/00220670903383069

Klassen, R. M., \& Chiu, M. M. (2010). Effects on teachers' self-efficacy and job satisfaction: Teacher gender, years of experience, and job stress. Journal of Educational Psychology, 102(3), 741-756. doi: 10.1037/a0019237

Klassen, R. M., Bong, M., Usher, E. L., Chong, W. H., Huan, V. S., Wong, I. Y. F., \& Georgiou, T. (2009). Exploring the validity of a teachers' self-efficacy scale in five countries. Contemporary Educational Psychology, 34(1), 67-76. doi: 10.1016/j.cedpsych.2008.08.001

Klassen, R. M., Foster, R.Y., Rajani, S., \& Bowman, C. (2009). Teaching in the Yukon: Exploring teachers' efficacy beliefs, stress, and job satisfaction in a remote setting. International Journal of Educational Research, 48(6), 381-394. doi: 10.1016/j.jjer.2010.04.002

Klassen, R. M., Usher, E. L., \& Bong, M. (2010). Teachers' collective efficacy, job satisfaction, and job stress in cross-cultural context. The Journal of Experimental Education, 78(4), 464-486. doi: $10.1080 / 00220970903292975$

Lent, R. W., \& Brown, S. D. (2008). Social cognitive career theory and subjective well-being in the context of work. Journal of Career Assessment, 16(1), 6-21. doi: 10.1177/1069072707305769

Lent, R. W., Nota, L., Soresi, S., Ginevra, M. C., Duffy, R. D., \& Brown, S. D. (2011). Predicting the job and life satisfaction of Italian teachers: Test of a social cognitive model. Journal of Vocational Behavior, 79(1), 91-97. doi: 10.1016/j.jvb.2010.12.006
Moè, A., Pazzaglia, F., \& Ronconi, L. (2010). When being able is not enough. The combined value of positive affect and self-efficacy for job satisfaction in teaching. Teaching and Teacher Education, 26(5), 1145-1153. doi: 10.1016/j.tate.2010.02.010

Organização para a Cooperação e Desenvolvimento Econômico (2014). Melhores competências, melhores empregos, melhores condições de vida: uma abordagem estratégica das políticas de competências, publicação da OCDE. doi: 10.1787/9789264177338-en.

Pinto, J. M. R., \& Alves, T. (2011). O impacto financeiro da ampliação da obrigatoriedade escolar no contexto do Fundeb. Educação e Realidade, 36, 605-624. Recuperado de http://seer.ufrgs.br/index. php/educacaoerealidade/article/view/15128/12931

Ramos, M. F. H. (2015). Modelo social cognitivo de satisfação no trabalho e eficácia coletiva: percepções sobre a docência (Tese de doutorado). Universidade Federal do Pará. Recuperado de http://ppgtpc.propesp.ufpa.br/index.php/br/teses-e-dissertacoes/teses/37-2015

Ramos, M. F. H., Costa, S. S., Pontes, F. A. R., Fernandez, A. P. O., \& Nina, K. C. F. (2014). Collective teacher efficacy beliefsf: A critical review of the literature. International Journal of Humanities and Social Science, 4(7), 179-188. Recuperado de http://www.ijhssnet.com/ journals/Vol_4_No_7_1_May_2014/23.pdf

Stephanou, G. (2013). The role of teachers' self- and collective-efficacy beliefs on their job satisfaction and experienced emotions in school. Psychology, 4(03), 268-278. doi: 10.4236/psych.2013.43A040

No artigo "Satisfação no trabalho docente: Uma análise a partir do modelo social cognitivo de satisfação no trabalho e da eficácia coletiva docente" publicado na edição 21(2):179-191 onde se lê: Amaély Ferreira Holanda Ramos, leia-se: Maély Ferreira Holanda Ramos 
Maély Ferreira Holanda Ramos, Doutora em Psicologia pelo Programa de Pós-graduação em Teoria e Pesquisa do Comportamento

(PPGTPC) da Universidade Federal do Pará (UFPA), é Pós-doutoranda em Psicologia no Programa de Pós-graduação em Teoria e Pesquisa do Comportamento (PPGTPC) da Universidade Federal do Pará (UFPA) e Professora da Faculdade de Educação, Instituto de Ciência da Educação, Universidade Federal do Pará (UFPA). Endereço para correspondência: Trav. Barão do Triunfo, Ed. Uno Tower, apto. 301, Bairro do Marco, CEP: 66.095-050, Belém (PA). Telefone: (91) 99284-8764. E-mail: maelyramos@ hotmail.com

Ana Patrícia de Oliveira Fernandez, Doutora em Psicologia pelo Programa de Pós-graduação em Teoria e Pesquisa do Comportamento (PPGTPC) da Universidade Federal do Pará (UFPA), é Professora no Instituto Federal de Educação, Ciência e Tecnologia do Pará (IFPA). E-mail: apsol2@hotmail.comutores

Karla Cristina Nina Furtado, Mestre em Psicologia pelo Programa de Pós-graduação em Teoria e Pesquisa do Comportamento (PPGTPC) da Universidade Federal do Pará (UFPA), é Professora no Instituto Federal de Educação, Ciência e Tecnologia do Pará (IFPA). E-mail: furtadokarla@hotmail.com

Edson Marcos Leal Soares Ramos, Doutor em Engenharia de Produção pela Universidade Federal de Santa Catarina (UFSC), é Professor Associado III da Universidade Federal do Pará (UFPA) e Professor Colaborador da Universidade de Cabo Verde (UNICV) no Mestrado de Segurança Pública. E-mail: ramosedson@gmail.com

Simone Souza da Costa e Silva, Doutora em Psicologia pela Universidade de Brasília (UnB), Pós-doutora em Ciências da Reabilitação pela Universidade de Dortmund, Alemanha, é Professora e Diretora Adjunta do Núcleo de Teoria e Pesquisa do Comportamento (NTPC) da Universidade Federal do Pará (UFPA). E-mail: symon.ufpa@gmail.com

Fernando Augusto Ramos Pontes, Doutor em Psicologia Experimental pela Universidade de São Paulo (USP), Pós-doutorado pela Universidade de Brasília e pela Technischen Universität Dortmund, Alemanha, é Professor Associado IV da Universidade Federal do Pará (UFPA), vinculado ao Núcleo de Teoria e Pesquisa do Comportamento (NTPC) e ao Programa de Pós-graduação em Teoria e Pesquisa do Comportamento (PPGTPC).E-mail: fernando.pontes@pesquisador.cnpq.br 